CLINICAL STUDY

\title{
Possible involvement of matrix metalloproteinase-3 in the pathogenesis of macroprolactinaemia in some patients with rheumatoid arthritis
}

\author{
Takashi Adachi, Naoki Hattori ${ }^{1}$, Takashi Ishihara ${ }^{2}$, Hirokazu Iida, Takanori Saito, Shigeo Miyashima ${ }^{3}$ and \\ Akira Shimatsu ${ }^{4}$ \\ Department of Orthopedics, Kansai Medical University, Osaka, Japan, ${ }^{1}$ Department of Pharmaceutical Sciences, Ritsumeikan University, \\ 1-1-1 Nojihigashi, Kusatsu-city, Shiga 525-8577, Japan, ${ }^{2}$ Department of Endocrinology, Kobe City General Hospital, Kobe, Japan, ${ }^{3}$ Miyashima RA \\ and Orthopedic Hospital, Osaka, Japan and ${ }^{4}$ Clinical Research Institute, National Hospital Organization Kyoto Medical Center, Kyoto, Japan \\ (Correspondence should be addressed to N Hattori; Email: hattorin@fc.ritsumei.ac.jp)
}

\begin{abstract}
Objective: Macroprolactin primarily comprises a complex of prolactin (PRL) and IgG molecules, particularly anti-PRL autoantibodies. However, it is unknown why autoantibodies against PRL develop in certain subjects. This study aimed to elucidate post-translational modifications in the PRL molecule that may be involved in the pathogenesis of macroprolactinaemia.

Methods: Macroprolactinaemia was screened with a polyethylene glycol method in 238 patients with rheumatoid arthritis (RA) and 302 control subjects and confirmed by gel chromatography. We examined the relationship between macroprolactinaemia and several RA-related laboratory tests including matrix metalloproteinase-3 (MMP-3) and anti-cyclic citrullinated peptide (CCP) antibody titres. The effect of MMP-3 on the PRL molecule was examined by western blotting.

Results: Patients with RA exhibited a significantly higher prevalence of macroprolactinaemia (15/238; $6.3 \%$ ) than the young control subjects (5/219 subjects; $2.3 \%)$, but the prevalence was not different from that observed in the elderly control subjects (5/83 subjects; $6.0 \%)$. The prevalence of macroprolactinaemia in patients with elevated MMP-3 levels $(9.68 \%)$ was significantly higher than that in those with normal MMP-3 levels (2.63\%). Digestion of PRL with MMP-3 produced vasoinhibins with several molecular species. Serum total and free PRL levels in RA patients were higher than those in the age- and gender-matched control subjects. The levels of macroprolactin were not significantly correlated with those of RA-specific anti-CCP antibody.

Conclusions: We speculate that elevated MMP-3 levels may lead to the formation of new epitopes on the PRL molecule that might trigger an immune response to produce anti-PRL autoantibodies in some patients with RA. Such post-translational modifications may possibly contribute to the increased prevalence of macroprolactinaemia in elderly subjects.
\end{abstract}

European Journal of Endocrinology 169 203-209

\section{Introduction}

Macroprolactinaemia is one of the major causes of hyperprolactinaemia $(1,2,3,4,5)$. In patients with macroprolactinaemia, the predominant form of prolactin (PRL) in the serum is macroprolactin, with a molecular mass $>150 \mathrm{kDa}$. The slow clearance of macroprolactin from the circulation, due to its large molecular size, is likely to cause hyperprolactinaemia (6). The prevalence of macroprolactinaemia has been reported to be $10-26 \%$ in patients with hyperprolactinaemia $(1,2,3,4)$ and $3.68 \%$ in the general population (5).

Macroprolactin is a heterogeneous molecule, but it mostly comprises PRL in a complex with IgG, in particular, with anti-PRL autoantibodies $(4,5,6,7,8,9)$.
However, it is not known why anti-PRL autoantibodies develop in certain subjects. In our previous study, we found that the prevalence of macroprolactinaemia was higher in aged subjects (5). Ageing is a progressive, degenerative process closely related to chronic inflammation; thus, with increasing age, more proteins are susceptible to post-translational modifications (10). Modifications in self-antigens that arise later in life may trigger reactions in the immune system. Autoantibodies against modified proteins may also cross-react with the original, unmodified proteins. We hypothesized that aberrant post-translational modifications in the PRL molecule could induce the production of anti-PRL autoantibodies.

Matrix metalloproteinase (MMP)-3 is an enzyme that degrades connective tissue matrix components, 
including proteoglycan, laminin, fibronectin and collagen $(11,12)$. MMP-3 is synthesized by synovial fibroblasts and chondrocytes, and its production is highly enhanced in rheumatoid arthritis (RA), a common autoimmune disorder. MMP-3 has a broad spectrum of substrate specificities, and PRL is one of its substrates (13). MMP-3 breaks down the PRL molecule into several fragments, known as vasoinhibins. Vasoinhibins comprise a family of peptides that suppress angiogenesis and promote apoptosis-mediated vascular regression (14). This post-translational modification of the PRL molecule may be involved in the induction of the production of anti-PRL autoantibodies.

In this study, we examined the relationship between macroprolactinaemia and MMP-3 and other inflammatory markers in patients with RA to clarify the mechanism underlying the development of autoantibodies against PRL.

\section{Subjects and methods}

\section{Subjects}

Macroprolactinaemia was screened in 238 patients with RA (200 women and 38 men, aged $60.7 \pm 12.5$ years (mean \pm s.D. )) who fulfilled the criteria for $\mathrm{RA}(15)$. We used 1987 ACR criteria for RA, not the 2010 ACR/EULAR classification, because most patients were diagnosed with RA before 2010. Serum samples were collected from 240 patients with RA, who gave informed consent to participate in the study from September 2011 to July 2012. Two patients were excluded from the study because they were taking domperidone, which could elevate serum PRL levels, for gastric symptoms. The disease duration of 238 patients with RA ranged from 0.3 to 44 years ( $13.9 \pm 7.2$ years). Their RA stages based upon the appearance of joints on the X-rays were as follows: 14 in stage I (no bone damage on X-rays), 16 in stage II (evidence of bone thinning around a joint), 112 in stage III (evidence of cartilage and bone damage), and 96 in stage IV (evidence of osteoporosis and ankylosis). The functional statuses of RA patients were as follows: 61 in class I (completely able to perform the usual activities of daily living), 118 in class II (limited performance in activities outside of work), 59 in class III (limited performance in work and other activities), and none in class IV (limited in the ability to perform usual self-care). These patients had received several combinations of the following drugs to suppress inflammation and immune reactions: biological agents such as infliximab $(n=93)$, methotrexate $(n=145)$, prednisolone $(n=107)$ and tacrolimus $(n=37)$; disease-modifying anti-rheumatic drugs such as salazosulphapyridine $(n=78)$; and nonsteroidal anti-inflammatory drugs such as diclofenac $(n=79)$. Control serum samples were acquired from 83 age- and gender-matched elderly control subjects
(69 women and 14 men, aged $59.0 \pm 2.4$ years) and 219 gender-matched young control subjects (185 women and 34 men, aged $29.4 \pm 2.6$ years) who were working at Kobe City General Hospital. The control subjects agreed to allow collecting extra sera than that drawn for their medical check-up to be used for this study, with the condition that only gender and age would be available as clinical information. Therefore, the possibility could not be ruled out that some control subjects might have RA.

Blood was drawn in the morning, and serum was separated and stored at $-80^{\circ} \mathrm{C}$ until use. This study was approved by the ethical committees of Kobe City General Hospital and Miyashima RA and Orthopaedic Hospital.

\section{Polyethylene glycol precipitation and PRL assays}

To determine free PRL concentrations, macroprolactin was removed from the serum samples $(50 \mu \mathrm{l})$ by mixing vigorously with $50 \mu \mathrm{l}$ cold polyethylene glycol (PEG; molecular weight $6000,25 \%$ in water) and centrifuging at $9100 \boldsymbol{g}$ for $10 \mathrm{~min}$. The supernatant was diluted tenfold with phosphate buffer $(0.01 \mathrm{M}, \mathrm{pH} 7.0)$ that contained $0.4 \mathrm{M} \mathrm{NaCl}$ and $0.1 \% \mathrm{BSA}$ (final serum dilution, 20-fold). To determine total PRL concentrations, additional serum samples were treated identically, but with water instead of PEG. Free PRL was defined as the PRL concentration in the supernatant after PEG precipitation; total PRL was defined as the PRL concentration in the water-treated sample. The PEG-precipitable PRL (\%), which represents the amount of macroprolactin, was calculated as follows: (total PRL - free PRL)/total PRL $\times 100$. When the amount of macroprolactin precipitated with PEG was $>60 \%$ (PRL recovery $<40 \%$ ), macroprolactinaemia was diagnosed.

PRL concentrations were measured in duplicate with an enzyme immunoassay for human PRL as described previously (5). In brief, serum samples were incubated with polystyrene balls (Precision Plastic Ball Co., Chicago, IL, USA) that were coated with anti-human PRL antiserum (NIDDK-Anti-human PRL IC-5) at $37^{\circ} \mathrm{C}$ for $6 \mathrm{~h}$. After washing, the balls were incubated at $4{ }^{\circ} \mathrm{C}$ overnight with affinity-purified, anti-human PRL Fab' fragments conjugated to HRP. This was followed by incubation at $20^{\circ} \mathrm{C}$ for $6 \mathrm{~h}$. After washing, the peroxidase activity bound to the balls was assayed in an enzyme reaction with 3-(p-hydroxyphenyl) propionic acid (Aldrich Chemical Co., Milwaukee, WI, USA) as the substrate. Results were evaluated by measuring fluorescence intensity with a spectrofluorophotometer (FP-6200ST; JASCO Co., Tokyo, Japan), with excitation at $320 \mathrm{~nm}$ and emission at $405 \mathrm{~nm}$.

Highly purified recombinant human PRL (human PRL-RP-2) and anti-human PRL polyclonal (rabbit) antibodies were provided by Dr A F Parlow of the Harbour-UCLA Medical Centre, National Hormone 
and Peptide Program, Torrance, CA, USA (parlow@ humc.edu). The upper reference value of serum total PRL was $525 \mathrm{mIU} / \mathrm{l}$ (conversion factor $=1 / 35$ for $\mathrm{mIU} / \mathrm{l}$ to $\mu \mathrm{g} / \mathrm{l})$. The limit of detection was $0.175 \mathrm{mIU} / \mathrm{l}$. The intra- and inter-assay coefficients of variation were 4 and $5 \%$ respectively, based on a serum sample with a total PRL concentration of $178.5 \mathrm{mIU} / \mathrm{l}$.

\section{Gel filtration chromatography}

Gel filtration chromatography was performed with a $1 \times 60 \mathrm{~cm}$ column of Ultrogel AcA 44 (IBF Biotechnics, Villeneuve La Garenne, France), equilibrated with $0.01 \mathrm{M}$ sodium phosphate buffer ( $\mathrm{pH} 7.0$ ), which contained $0.1 \mathrm{M} \mathrm{NaCl}, 0.1 \% \mathrm{BSA}$ and $0.01 \% \mathrm{NaN}_{3}$. Serum samples $(100 \mu \mathrm{l})$ were applied to the column, and $1 \mathrm{ml}$ fractions were collected for the determination of PRL concentrations. The column was calibrated with various molecular weight markers (Sigma).

\section{Biochemical markers}

Serum levels of MMP-3 were measured with Panaclear MMP-3 (Latex; Sekisui Medical Co., Tokyo, Japan). The reference intervals were $36.9-121.0 \mathrm{ng} / \mathrm{ml}$ for men and $17.3-59.7 \mathrm{ng} / \mathrm{ml}$ for women. Values greater than the maximum reference value were categorized as abnormally high.

Serum levels of C-reactive protein (CRP) were measured with an ELISA (LZ test Eiken CRP-HG; Eiken Kagaku, Tokyo, Japan), according to the manufacturer's instructions. Values $>0.3 \mathrm{mg} / \mathrm{dl}$ were categorized as abnormally high.

The erythrocyte sedimentation ratio (ESR) was measured by Falco Biosystems (Kyoto, Japan). Values $>10 \mathrm{~mm} / \mathrm{h}$ for men and $>15 \mathrm{~mm} / \mathrm{h}$ for women were categorized as abnormally high.

The PEG-precipitable protein ratio (\%), which represents the amount of $\gamma$-globulin plus proteins that bind to PEG nonspecifically, was calculated as follows: (total protein-protein in the supernatant after treatment with $12.5 \%$ PEG)/total PRL $\times 100$. The protein concentrations were measured with a Micro BCA protein assay kit (Pierce Biotechnology, Rockford, IL, USA), according to the manufacturer's instructions.

Serum levels of antibodies against cyclic citrullinated peptides (anti-CCP) were measured with Immunoscan CCPlus (Euro Diagnostica, Malmo, Sweden), according to the manufacturer's instructions. Samples with more than $25 \mathrm{U} / \mathrm{ml}$ of anti-CCP antibodies were defined as positive.

\section{Digestion of PRL with MMP-3}

MMP-3 was activated according to the manufacturer's instructions. Recombinant human MMP-3 (R\&D Systems, Inc., Minneapolis, MN, USA) was activated at
$20 \mu \mathrm{g} / \mathrm{ml}$ in an assay buffer (50 mM Tris, $10 \mathrm{mM} \mathrm{CaCl}_{2}$, $150 \mathrm{mM} \mathrm{NaCl}$ and $0.05 \%$ Brij-35, pH 7.5) containing $5 \mu \mathrm{g} / \mathrm{ml}$ chymotrypsin. The mixture was incubated at $37^{\circ} \mathrm{C}$ for $30 \mathrm{~min}$, and the reaction was stopped by adding $2 \mathrm{mM}$ phenymethylsulphonyl fluoride (PMSF). PRL (50 ng) was incubated with various amounts of the activated MMP-3 in $10 \mu \mathrm{l}$ assay buffer at $37^{\circ} \mathrm{C}$ for $1 \mathrm{~h}$ and subjected to SDS-PAGE and western blotting.

\section{SDS-PAGE and western blotting}

MMP-3-digested PRL was heated at $100{ }^{\circ} \mathrm{C}$ in $125 \mathrm{mM}$ Tris-HCl buffer ( $\mathrm{pH} 8.3$ ) containing $4 \mathrm{M}$ urea, $2 \%$ SDS and 5\% 2-mercaptoethanol for $3 \mathrm{~min}$ and electrophoresed on a $10 \%$ polyacrylamide gel containing $0.1 \%$ SDS. The proteins in the gel were electrotransferred onto a PVDF membrane (Bio-Rad Laboratories, Inc.) and incubated with anti-human PRL antiserum. After incubation with goat anti-rabbit immunoglobulin-peroxidase conjugate (Cosmo Bio, Co., Tokyo, Japan), the bands were visualized with ECL western blotting detection reagents (GE Healthcare, Buckinghamshire, UK) and detected by a lumino-image analyzer (LAS-4000 mini, Fuji Photo Film Co., Ltd., Kanagawa, Japan).

\section{Statistical analysis}

Values are expressed as means \pm s.D. Statistical significance was evaluated with Student's unpaired t-test and the $\chi^{2}$ test in StatMate III statistic software (ATMS, Tokyo, Japan). Correlation coefficients between variables were calculated by a linear regression analysis. Serum PRL concentrations and anti-CCP antibodies

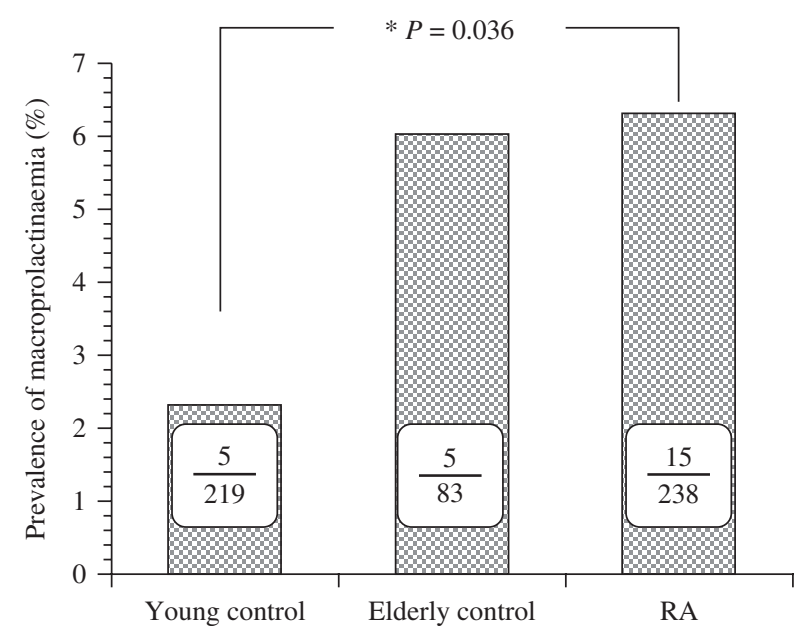

Figure 1 Prevalence of macroprolactinaemia in patients with RA and control subjects. The prevalence of macroprolactinaemia is shown for young control subjects, elderly control subjects and patients with RA. The numbers shown in the bars indicate the number of subjects with macroprolactinaemia/total number of subjects in that group. The $\chi^{2}$ test was used to compare the groups. 
Table 1 Clinical characteristics and parameters of the study subjects.

\begin{tabular}{lccc}
\hline & Young control & Elderly control & RA \\
\hline Number of subjects & 219 & 83 & 238 \\
Women/men & $185 / 34$ & $69 / 14$ & $200 / 38$ \\
Age & $29.4 \pm 2.6$ & $59.4 \pm 2.4$ & $60.7 \pm 12.5$ \\
Total PRL $(\mathrm{mlU} / \mathrm{l})$ & $233.4(63.0-882.0)$ & $113.1(45.5-273.0)$ & $225.4^{*}(77.0-654.5)$ \\
Free PRL $(\mathrm{mlU} / \mathrm{l})$ & $149.1(42.0-514.5)$ & $73.2(35.0-147.0)$ & $141.8^{\dagger}(56.0-353.5)$ \\
MMP-3 $(\mathrm{ng} / \mathrm{ml})$ & $49.8 \pm 18.9$ & $56.6 \pm 21.8^{\ddagger}$ & $120.6 \pm 88.2^{\S}$ \\
ESR $(\mathrm{mm} / \mathrm{h})$ & $\mathrm{ND}$ & $\mathrm{ND}$ & $27.0 \pm 18.7$ \\
CRP $(\mathrm{mg} / \mathrm{dl})$ & $\mathrm{ND}$ & $\mathrm{ND}$ & $0.76 \pm 0.87$ \\
Anti-CCP antibody $(\mathrm{U} / \mathrm{ml})$ & $\mathrm{ND}$ & $\mathrm{ND}$ & $293.4(14.5-6023.3)$ \\
\hline
\end{tabular}

${ }^{*}$ Mean $(95 \% \mathrm{Cl}) . P<0.001$ compared with that of the age- and gender-matched elderly control subjects. ${ }^{\dagger} P<0.001$ compared with that of the age- and gender-matched elderly control subjects. ${ }^{\ddagger} P=0.045$ compared with that of the young control subjects. ${ }^{\S} P<0.001$ compared with that of the young and elderly control subjects.

were logarithmically transformed to get the distribution close to normal. The 95\% CIs for ln-transformed data were determined and back-transformed. $P<0.05$ was considered significant.

\section{Results}

\section{Prevalence of macroprolactinaemia in $R A$}

Fifteen patients with RA had macroprolactinaemia (15/238 patients; $6.3 \%)$. The prevalence among patients with RA was significantly $(P=0.036)$ higher than that in the gender-matched young control group (5/219 subjects; $2.3 \%)$, but not different $(P=0.93)$ from that in the age- and gender-matched elderly control group (5/83 subjects; 6.0\%) (Fig. 1).

Serum total PRL concentrations were significantly higher in patients with RA (mean: $225.4 \mathrm{mIU} / \mathrm{l}, 95 \%$ CI: 77.0-654.5 mIU/l) than in the age- and gendermatched control subjects $(113.1 \mathrm{mIU} /, \quad 45.5-$ $273.0 \mathrm{mIU} / \mathrm{l} ; \mathrm{P}<0.001$; Table 1). Serum free PRL concentrations were also significantly higher in patients with RA (141.8 mIU/l, 56.0-353.5 mIU/l) than in the age- and gender-matched control subjects $(73.2 \mathrm{mIU} / \mathrm{l}$, 35.0-147.0 mIU/l; $P<0.001$; Table 1). Hyperprolactinaemia (serum total PRL concentrations $>525 \mathrm{mIU} / \mathrm{l}$ ) occurred more frequently in RA patients $(26 / 238$ patients; $10.9 \%$ ) than in the elderly control subjects (2/83 patients; $2.4 \% ; P=0.018$ ).

We examined the levels of three indicators of RA disease activity: MMP-3, CRP and ESR. Increases in the levels of these markers reflect greater severity of inflammation and joint destruction. We found that serum MMP-3 levels were elevated in 124 patients (52.1\%) with RA. Patients with elevated MMP-3 levels had a significantly higher prevalence of macroprolactinaemia $(12 / 124$ patients; $9.68 \% ; P=0.025)$ than those with normal MMP-3 levels (3/114 patients; $2.63 \%$; Fig. 2). As shown in Table 1, serum MMP-3 levels in the elderly control subjects $(56.6 \pm 21.8 \mathrm{ng} / \mathrm{ml})$ were significantly $(P=0.045)$ higher than those in the young control subjects $(49.8 \pm 18.9 \mathrm{ng} / \mathrm{ml})$. Serum CRP and ESR levels were elevated in 107 and 142 patients respectively. However, the prevalence of macroprolactinaemia was not significantly different between patients with and without elevations in the levels of these inflammatory markers (Fig. 2).

\section{Digestion of PRL with MMP-3}

PRL (50 ng) was digested with various amounts of MMP-3 (1, 2, 4, 8 and $20 \mathrm{ng}$ ) in $10 \mu \mathrm{l}$ assay buffer. As shown in Fig. 3, digestion of the $23 \mathrm{kDa}$ PRL molecule with MMP-3 produced 14, 15 and $16 \mathrm{kDa}$ vasoinhibins. As the amount of MMP-3 increased, the proportion of larger vasoinhibin species decreased and that of smaller species increased. The $23 \mathrm{kDa}$ PRL band migrated a little slowly when treated with MMP-3 with the cause being unknown.

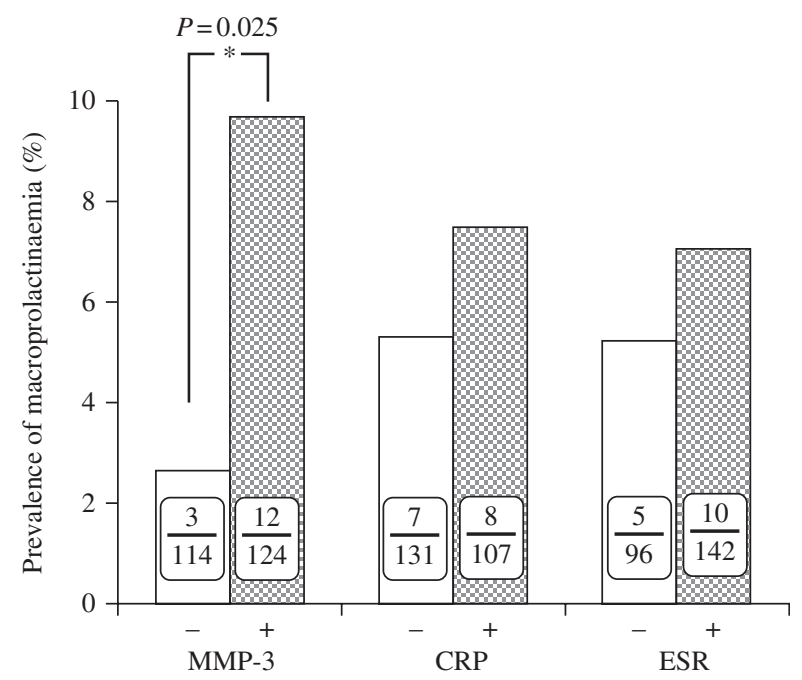

Figure 2 Prevalence of macroprolactinaemia in patients with RA who exhibited high or low levels of inflammatory markers. The prevalence of macroprolactinaemia was examined in patients with RA who exhibited high $(+)$ or low $(-)$ disease activity, based on serum levels of MMP-3, CRP and ESR. The numbers shown in the bars indicate the number of patients with macroprolactinaemia/total number of patients in that group. The $\chi^{2}$ test was used to compare the groups. 


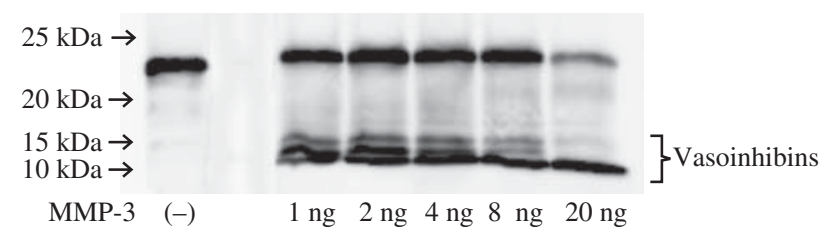

Figure 3 Digestion of PRL with MMP-3. PRL $(50 \mathrm{ng})$ was digested with various amounts of MMP-3 (1, 2, 4, 8 and $20 \mathrm{ng})$ in $10 \mu \mathrm{l}$ assay buffer and analysed by SDS-PAGE and western blotting. The lane $(-)$ indicates PRL incubated in the absence of MMP-3. The positions of the molecular weight markers are indicated by arrows.

\section{Relationship between macroprolactin and $\gamma$-globulin and anti-CCP antibody}

The levels of PEG-precipitable protein, which includes $\gamma$-globulin and proteins that bind to PEG nonspecifically, ranged from 14.3 to $55.0 \%(32.5 \pm 7.5 \%)$ in patients with RA. The levels of PEG-precipitable protein were not significantly correlated with those of the PEG-precipitable PRL ( $r=0.02$; Fig. 4a). The levels of PEG-precipitable protein were not significantly different between RA patients with and without macroprolactinaemia $(30.3 \pm 8.6$ and $32.6 \pm 7.5 \%$ respectively, $P=0.26$ ). Gel filtration chromatography confirmed that all 15 patients with RA and macroprolactinaemia had significantly elevated ratios of PRL molecules larger than $150 \mathrm{kDa}$ (macroprolactin; $59.7 \pm 21.5 \%$, range $26.9-94.2 \%$ ) than those with RA but without macroprolactinaemia $(n=10 ; 2.9 \pm$ $2.3 \%$, range $0-7.1 \%, P<0.001$; Fig. $4 b$ ). Anti-CCP antibody was positive $(>25 \mathrm{U} / \mathrm{ml})$ in 212 of 238 (89.1\%) patients with RA. However, there was no significant correlation between the levels of anti-CCP antibody and the levels of PEG-precipitable PRL $(r=0.09$; Fig. $4 \mathrm{c})$.

\section{Discussion}

Macroprolactin is a heterogeneous molecule, but it mainly comprises a complex of PRL with IgG, particularly anti-PRL autoantibodies. The results of this study suggest that elevated levels of MMP-3 might contribute to the generation of anti-PRL autoantibodies in some patients with RA.

MMP-3 is an enzyme secreted by synovial fibroblasts and chondrocytes that degrades connective tissue matrix components. Increased levels of MMP-3 in serum and synovial fluids are frequently observed in RA. MMP-3 levels reportedly reflect the intensity of intrasynovial inflammation and joint destruction $(11,12)$. MMP-3 has a broad spectrum of substrate specificities, and PRL is one of its substrates. MMP-3 cleaves the PRL molecule at Leucine ${ }^{156}$-Glutamine ${ }^{157}$ and at Alanine $^{111}$-Isoleucine ${ }^{112}$. This produces N-terminal fragments of 17 and $12 \mathrm{kDa}$ respectively (13). These $\mathrm{N}$-terminal fragments of the PRL molecule are termed 'vasoinhibins' because they inhibit angiogenesis by suppressing growth factor-induced endothelial cell proliferation (14). In contrast, the parental $23 \mathrm{kDa}$ PRL molecule lacks such an effect on endothelial cells. In this study, we confirmed that MMP-3 could digest the PRL molecule and produce vasoinhibins with various molecular species. We tried to identify the presence of vasoinhibins in the sera collected from (a)
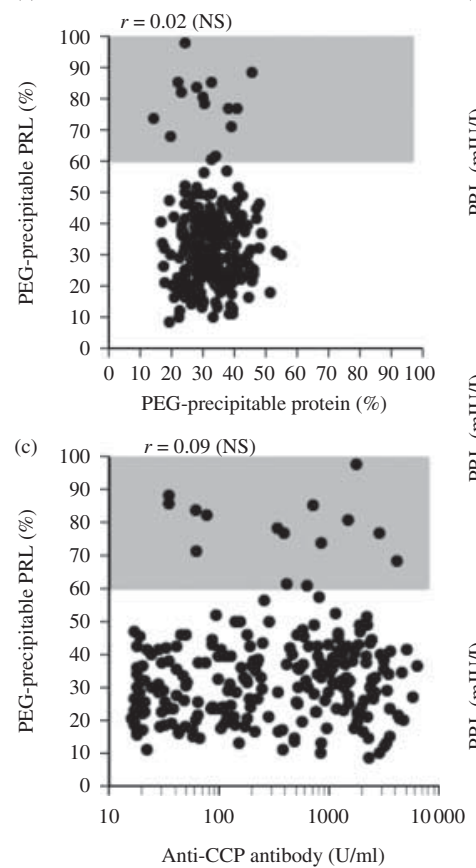

(b)
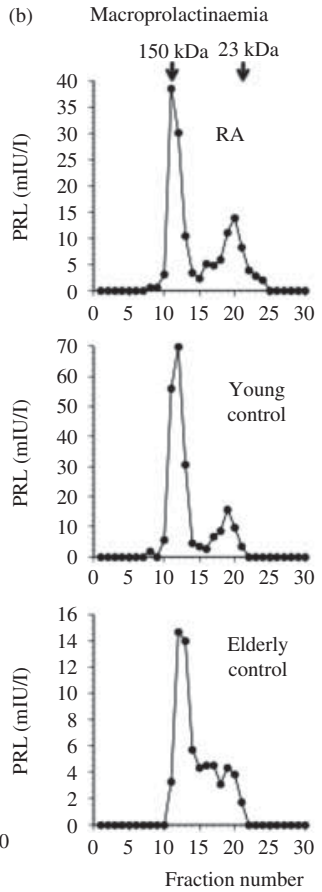

Non-macroprolactinaemia
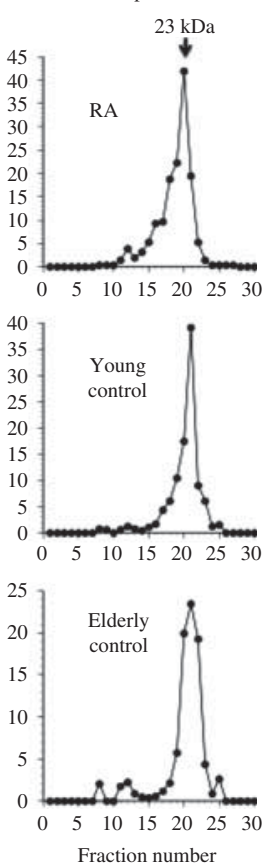

Figure 4 Relationship between the levels of macroprolactin and those of $\gamma$-globulin and anti-CCP antibody. (a) Correlation between the ratios of the PEG-precipitable protein $(\gamma$-globulin) and PEG-precipitable PRL (macroprolactin) in patients with RA. Macroprolactinaemia is indicated by the shaded area (PEG-precipitable PRL ratio $>60 \%$ ). (b) Representative gel filtration chromatographic profiles of serum PRL of RA patients, young control subjects, and elderly control subjects with and without macroprolactinaemia. Macroprolactin is represented by the $150 \mathrm{kDa}$ peak and monomeric $\mathrm{PRL}$ is represented by the $23 \mathrm{kDa}$ peak (c) Correlation between the levels of anti-CCP antibody and PEG-precipitable PRL (macroprolactin) in patients with RA Macroprolactinaemia is indicated by the shaded area (PEG-precipitable PRL ratio $>60 \%$ ). 
RA patients with elevated MMP-3 levels. However, we were unable to obtain definitive results because the volume of the clinical samples was not sufficient and the serum PRL levels were not high enough to be detected by western blotting.

The present finding that the prevalence of macroprolactinaemia in RA patients with elevated serum MMP-3 levels was higher than that in those with normal levels might indicate that MMP-3 is involved in the pathogenesis of macroprolactinaemia in some RA patients. The cleavage of the PRL molecule may expose new epitopes that elicit an immune response and production of anti-PRL autoantibodies. These autoantibodies may cross-react with the parental PRL molecule and generate macroprolactin. However, it is unlikely that MMP-3 is an independent cause of macroprolactinaemia, in general, because the prevalence of macroprolactinaemia in RA patients was higher than that in the young control subjects but similar to that in the age- and gender-matched control subjects. If MMP-3 is an independent cause of macroprolactinaemia, the effect of elevated MMP-3 levels should be added to that of ageing, resulting in a higher prevalence of macroprolactinaemia in RA patients than in the age-matched control subjects. Serum MMP-3 levels in the elderly control subjects were found to be higher than those in the young control subjects, in accordance with the previous report that serum MMP-3 values were positively correlated with age (16). Some unknown factor operating upstream of MMP-3 and ageing may be involved in the pathogenesis of macroprolactinaemia.

Macroprolactinaemia screening is performed with $12.5 \%$ PEG, which precipitates $\gamma$-globulin. One study has shown that some patients with high serum $\gamma$-globulin levels had pseudomacroprolactinaemia; thus, no macroprolactin was observed on gel chromatography, despite an increase in the PEG-precipitable PRL ratio (17). Because RA is a chronic inflammatory disorder characterized by elevated $\gamma$-globulin levels, some patients with RA might be expected to exhibit pseudomacroprolactinaemia. However, in the present study, we did not observe any difference in the PEG-precipitable protein $(\gamma$-globulin plus proteins that bind to PEG nonspecifically) levels between patients with and without macroprolactinaemia. Furthermore, there was no correlation between the PEG-precipitable protein and PEG-precipitable PRL levels in our patients. Moreover, we confirmed that all 15 patients with RA and macroprolactinaemia (judged by the PEG method) actually had macroprolactinaemia, based on gel chromatography. This suggests that such levels of $\gamma$-globulin present in RA may not directly cause pseudomacroprolactinaemia.

Several studies have shown that serum PRL levels in patients with RA are elevated $(18,19)$. The present study demonstrated that serum total and free PRL levels in RA patients were significantly higher than those in the age- and gender-matched control subjects. Since PRL reportedly induces the maturation of $\mathrm{T}$ cells, develops antigen-presenting cells, enhances immunoglobulin production and breaks down B-cell tolerance to self via its anti-apoptotic action (20), the increase in serum PRL levels may contribute to the development of RA.

In the early literature on macroprolactinaemia, some associations have been reported between macroprolactinaemia and autoimmune disorders $(21,22)$. However, later studies that included larger patient cohorts have revealed that, although many patients with macroprolactinaemia possessed antithyroglobulin and thyroid peroxidase antibodies, the prevalence of these autoantibodies was not significantly different from that observed in the controls $(3,4,9)$. Wallace et al. (23) followed 51 patients with macroprolactinaemia for 10 years, and they did not observe any symptoms suggestive of the development of autoimmune conditions. Anti-CCP antibody is a specific autoantibody in RA, and the titre of anti-CCP antibody is related to RA severity (24). In the present study, we found no correlation between the ratios of macroprolactin and the levels of anti-CCP antibodies. The results of the present study indicate that the development of anti-PRL autoantibodies might not be associated with that of RA-specific antibodies.

In summary, our findings indicate a possibility that MMP-3 might be involved in the development of macroprolactinaemia in some patients with RA and that such post-translational modifications of the PRL molecule may lead to the increased prevalence of macroprolactinaemia in elderly subjects.

\section{Declaration of interest}

The authors declare that there is no conflict of interest that could be perceived as prejudicing the impartiality of the research reported.

\section{Funding}

This work was supported by the Domestic Fund of Ritsumeikan University.

\section{Acknowledgements}

We thank Dr A F Parlow, scientific director of the National Hormone and Pituitary Program, for supplying PRL and the antisera. We also thank Geoff Gillespie for his assistance in preparing this manuscript.

\section{References}

1 Bjøro T, Mørkrid L, Wergeland R, Turter A, Kvistborg A, Sand T \& Torjesen P. Frequency of hyperprolactinaemia due to large molecular weight prolactin (150-170 kD PRL). Scandinavian Journal of Clinical and Laboratory Investigation 199555 139-147. (doi:10.3109/00365519509089606)

2 Leslie H, Courtney CH, Bell PM, Hadden DR, McCance DR, Ellis PK, Sheridan B \& Atkinson AB. Laboratory and clinical experience in 55 patients with macroprolactinemia identified by a simple polyethylene glycol precipitation method. Journal of Clinical Endocrinology and Metabolism 200186 2743-2746. (doi:10.1210/ jc.86.6.2743) 
3 Vallette-Kasic S, Morange-Ramos I, Selim A, Gunz G, Morange S, Enjalbert A, Martin PM, Jaquet P \& Brue T. Macroprolactinemia revisited: a study on 106 patients. Journal of Clinical Endocrinology and Metabolism 200287 581-588. (doi:10.1210/jc.87.2.581)

4 Gibney J, Smith TP \& McKenna TJ. Clinical relevance of macroprolactin. Clinical Endocrinology 200562 633-643. (doi:10.1111/ j.1365-2265.2005.02243.x)

5 Hattori N, Ishihara T \& Saiki Y. Macroprolactinaemia: prevalence and aetiologies in a large group of hospital workers. Clinical Endocrinology $2009 \mathbf{7 1}$ 702-708. (doi:10.1111/j.1365-2265. 2009.03570.x)

6 Hattori N \& Inagaki C. Anti-prolactin (PRL) autoantibodies cause asymptomatic hyperprolactinemia: bioassay and clearance studies of PRL-immunoglobulin G complex. Journal of Clinical Endocrinology and Metabolism $1997 \mathbf{8 2}$ 3107-3110. (doi:10.1210/jc.82.9.3107)

7 Hattori N, Ishihara T, Ikekubo K, Moridera K, Hino M \& Kurahachi H. Autoantibody to human prolactin in patients with idiopathic hyperprolactinemia. Journal of Clinical Endocrinology and Metabolism 199275 1226-1229. (doi:10.1210/jc.75.5.1226)

8 De Schepper J, Schiettecatte J, Velkeniers B, Blumenfeld Z, Shteinberg M, Devroey P, Anckaert E, Smitz J, Verdood P, Hooghe $\mathrm{R}$ et al. Clinical and biological characterization of macroprolactinemia with and without prolactin-IgG complexes. European Journal of Endocrinology $2003 \quad 149$ 201-207. (doi:10.1530/eje.0.1490201)

9 Kavanagh-Wright L, Smith TP, Gibney J \& McKenna TJ. Characterization of macroprolactin and assessment of markers of autoimmunity in macroprolactinaemic patients. Clinical Endocrinology 2009 70 599-605. (doi:10.1111/j.1365-2265. 2008.03402.x)

10 Cloos PA \& Christgau S. Post-translational modifications of proteins: implications for aging, antigen recognition, and autoimmunity. Biogerontology 20045 139-158. (doi:10.1023/B:BGEN.00000 31152.31352.8b)

11 So A, Chamot AM, Péclat V \& Gerster JC. Serum MMP-3 in rheumatoid arthritis: correlation with systemic inflammation but not with erosive status. Rheumatology 199938 407-410. (doi:10.1093/rheumatology/38.5.407)

12 Green MJ, Gough AK, Devlin J, Smith J, Astin P, Taylor D \& Emery P. Serum MMP-3 and MMP-1 and progression of joint damage in early rheumatoid arthritis. Rheumatology $2003 \mathbf{4 2}$ 83-88. (doi:10.1093/rheumatology/keg037)

13 Macotela Y, Aguilar MB, Guzmán-Morales J, Rivera JC, Zermeño C, López-Barrera F, Nava G, Lavalle C, Martínez de la Escalera G \& Clapp C. Matrix metalloproteases from chondrocytes generate an antiangiogenic 16 kDa prolactin. Journal of Cell Science 2006119 1790-1800. (doi:10.1242/jcs.02887)
14 Clapp C, Aranda J, González C, Jeziorski MC \& Martínez de la Escalera G. Vasoinhibins: endogenous regulators of angiogenesis and vascular function. Trends in Endocrinology and Metabolism 200617 301-307. (doi:10.1016/j.tem.2006.08.002)

15 Arnett FC, Steven M, Edworthy SM, Bloch DA, Mcshane DJ, Fries JF, Cooper NS, Healey LA, Kaplan SR, Liang MH et al. The American Rheumatism Association 1987 revised criteria for the classification of rheumatoid arthritis. Arthritis and Rheumatism 198831 315-324. (doi:10.1002/art.1780310302)

16 Komosinska-Vassev K, Olczyk P, Winsz-Szczotka K, KuznikTrocha K, Klimek K \& Olczyk K. Age- and gender-dependent changes in connective tissue remodeling: physiological differences in circulating MMP-3, MMP-10, TIMP-1 and TIMP-2 level. Gerontology 201157 44-52. (doi:10.1159/000295775)

17 Ram S, Harris B, Fernando JJ, Gama R \& Fahie-Wilson M. Falsepositive polyethylene glycol precipitation tests for macroprolactin due to increased serum globulins. Annals of Clinical Biochemistry 200845 256-259. (doi:10.1258/acb.2008.007233)

18 Mateo L, Nolla JM, Bonnin MR, Navarro MA \& Roig-Escofet D. High serum prolactin levels in men with rheumatoid arthritis. Journal of Rheumatology 199825 2077-2082.

19 Fojtíková M, Tomasová Studýnková J, Filková M, Lacinová Z, Gatterová J, Pavelka K, Vencovský J \& Senolt L. Elevated prolactin levels in patients with rheumatoid arthritis: association with disease activity and structural damage. Clinical and Experimental Rheumatology $2010 \mathbf{2 8} 849-854$.

20 Jara LJ, Medina G, Saavedra MA, Vera-Lastra O \& Navarro C. Prolactin and autoimmunity. Clinical Reviews in Allergy $\mathcal{E}$ Immunology 2011 40 50-59. (doi:10.1007/s12016-009-8185-3)

21 Malarkey WB, Jackson R \& Wortsman J. Long-term assessment of patients with macroprolactinemia. Fertility and Sterility $1988 \mathbf{5 0}$ 413-418.

22 Leite V, Cosby H, Sobrinho LG, Fresnoza MA, Santos MA \& Friesen HG. Characterization of big, big prolactin in patients with hyperprolactinaemia. Clinical Endocrinology 199237 365-372. (doi:10.1111/j.1365-2265.1992.tb02340.x)

23 Wallace IR, Satti N, Courtney CH, Leslie H, Bell PM, Hunter SJ, McCance DR, Sheridan B \& Atkinson AB. Ten-year clinical followup of a cohort of 51 patients with macroprolactinemia establishes it as a benign variant. Journal of Clinical Endocrinology and Metabolism 2010 95 3268-3271. (doi:10.1210/jc.2010-0114)

24 Bizzaro N, Mazzanti G, Tonutti E, Villalta D \& Tozzoli R. Diagnostic accuracy of the anti-citrulline antibody assay for rheumatoid arthritis. Clinical Chemistry 200147 1089-1093.

Received 28 February 2013

Revised version received 12 May 2013

Accepted 29 May 2013 